\title{
Grands projets de ville : un lieu de production symbolique du territoire
}

Major Cities Projects Program: A Place of Symbolic Production of the Territory

\section{Stefan Bratosin}

\section{(2) OpenEdition}

Journals

Édition électronique

URL : http://journals.openedition.org/edc/103

DOI : $10.4000 /$ edc. 103

ISSN : 2101-0366

Éditeur

Université Lille-3

Édition imprimée

Date de publication : 1 décembre 2003

ISSN : 1270-6841

Référence électronique

Stefan Bratosin, «Grands projets de ville : un lieu de production symbolique du territoire », Études de communication [En ligne], 26 | 2003, mis en ligne le 10 octobre 2008, consulté le 01 mai 2019. URL: http://journals.openedition.org/edc/103; DOI : 10.4000/edc.103

Ce document a été généré automatiquement le 1 mai 2019.

(c) Tous droits réservés 


\title{
Grands projets de ville : un lieu de production symbolique du territoire
}

Major Cities Projects Program: A Place of Symbolic Production of the Territory

\author{
Stefan Bratosin
}

1 La portée de la notion de territoire employée ici relève de l'approche géographique de l'espace uniquement dans la mesure où elle est indispensable pour saisir le déploiement de la projection symbolique d'une ou des organisation(s) spécifique(s) - juridiques, politiques, économiques, sociales, etc. - sur un espace donné. Il n'est pas question cependant d'identifier et de traiter séparément ces organisations, mais de le faire dans leur globalité, notamment en interrogeant la symbolique politique issue de leur investissement collectif sans rechercher des distinctions particulières en termes d'agents. Dès lors, dans l'étude suivante, la notion de territoire fait référence, certes, à la construction complexe de nature juridique, sociale et culturelle avec laquelle les géographes opèrent d'ordinaire, mais elle s'attache à expliciter seulement le rôle des lieux de production symbolique dans le processus actuel de renouvellement urbain, lorsque les territoires de la ville sont rattachés à un programme politique, en l'occurrence celui des Grands Projets de Ville (GPV).

\section{Les territoires GPV : références et productions symboliques}

2 L'intervention politique sur les territoires de la ville est avant tout un processus de symbolisation. Les deux aspects de ce processus de symbolisation sont la référence au symbolique et la production du symbolique. La référence au symbolique est le moteur dans la perspective de l'aménagement des territoires. Elle comporte deux versants :

L'opération symbolique sur le modèle de la communication eucharistique et à quoi correspondent les grands moments ou mouvements de fusion, et les images symboliques, polysémiques, pièges à flux qui condensent en elles, en surimpression les attentes les plus contradictoires (Sfez, 1992, p. 461). 
3 La référence au symbolique est une présence dominante et illustrative déjà de l'aménagement des territoires dans le programme des Grands Projets Urbains (GPU). Lancé entre 1991 et 1994 sur la base de décisions du Comité Interministériel des Villes (CIV), le programme des GPU a été conçu initialement pour répondre à des dynamiques de déclin que les instruments traditionnels de la politique de la ville ne permettaient pas d'enrayer. Il concernait une quinzaine de sites frappés par une symbolique dévalorisante en termes d'espace, mais aussi en ce qui concerne l'économie, le social et l'image. Il visait dans l'espace urbain, le grand ensemble isolé des autres quartiers de la ville, renfermé sur lui-même, mono-fonctionnel, mal desservi par les transports en commun, lieu de vie caractérisé par le fort taux de chômage et de délinquance, le quartier rejeté par les entreprises et ayant une mauvaise réputation sur le marché de l'habitat. L'objectif des GPU était alors de réinsérer le quartier dans son agglomération, c'est-à-dire de

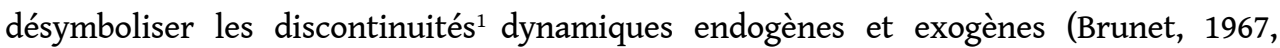
pp. 35-36) en s'appuyant sur des opérations de transformation urbaine inscrites dans la durée. Dans cette logique, la réussite des projets exigeait des opérations conjointes d'urbanisme et de reconstruction des références symboliques. Mais cela ne fut pas toujours le cas à cause de la prise en compte quasi unanime principalement du sens du territoire issu de la perspective d'urbanisme accroché au bornage physique, ancré au sol (Webber, 1996). Ainsi, le sens des territoires concernés par les GPU est construit avec prédilection par rapport à l'isolement matériel d'une partie de la ville. Les territoires à "transformer en profondeur $»^{2}$ sont, par exemple, des centres commerciaux, faisant fonction de centralité urbaine, conçus comme des objets fermés, introvertis, et qui ne donnent à voir à la ville que leurs façades aveugles, voire leurs parkings et leurs quais de déchargement. Il s'agit des territoires participant d'urbanisation de dalles et de coursives, séparées de la circulation des voitures, sans rapport à la rue, qui forment des entrelacements de circulation complexes, peu lisibles, et insécurisés. Ces territoires sont des îlots urbains "forteresses", voire des quartiers entiers qui sont en quelque sorte " réservés ", sans osmose possible avec leur environnement, difficiles à traverser, incitant au contournement soit par la forme des bâtiments, soit par la conception en cul-de-sac, soit par l'illisibilité des voies et des cheminements générateurs d'un sentiment d'insécurité. Ils se structurent autour d'unités monolithiques où il est excessivement difficile de changer une partie sans mettre en cause l'ensemble. Par conséquent, la transformation en profondeur recherchée au départ en termes de projet est en décalage avec la déclinaison sur le terrain de l'action publique. Alors qu'il était initialement question d'une opération favorisant les « appropriations tacites » (Gay, 1995, pp. 40-42) de certains espaces urbains, l'action débouche sur une course à l'aménagement afin d'augmenter la capacité d'attraction de ces espaces. La reconquête des territoires concernés par les GPU exploitera donc la référence au symbolique ${ }^{3}$ articulée dans les meilleurs des cas autour des résorptions de friches par la réalisation de parcs urbains ou d'équipements sportifs, autour des réhabilitations de bâtiments industriels, emblématiques de l'histoire de la ville, transformés en équipements sociaux, de loisirs, culturels ou économiques, autour des réalisations d'équipements de quartiers et de pôles de services publics, autour des interventions sur la voirie, les rez-de-chaussée commerciaux, les logements, etc.

4 La production du symbolique, l'autre aspect du processus de symbolisation dont participe l'intervention politique sur la ville, apparait alors comme la condition complémentaire sine qua non pour le renouvellement urbain. Sa présence dans le déploiement du 
programme GPV est marquée par la réactivation des dispositifs visant la « condensation dans un seul point des fragments épars, contradictoires, voire incompatibles » (Sfez, 1992, p. 471). Ainsi, dans le cadre du plan gouvernemental de rénovation urbaine et de solidarité, lorsque le CIV décide en décembre 1999 de mettre en œuvre un programme de renouvellement urbain centré autour des GPV, la mutation opérée est avant tout au niveau de la projection symbolique de l'action politique sur les territoires concernés. La préoccupation pour la production symbolique l'emporte sur celle de la référence au symbolique. Un GPV, tout comme un GPU, vise à réinsérer les quartiers dans l'agglomération. Il permet la mise en œuvre d'opérations de requalification urbaine, en mobilisant notamment des capacités d'étude et d'investissement. Cependant, le GPV se distingue du GPU, car il est un projet global de développement social et urbain, intégré au contrat de ville. Le contrat de ville constitue désormais un cadre dans lequel les différents partenaires s'engagent à mettre en œuvre, de façon concertée, des politiques territorialisées de développement solidaire et de renouvellement urbain. Il a pour objectif le développement équilibré de la ville permettant l'intégration harmonieuse de toutes ses composantes. Le programme GPV apporte, dans ce cadre, pour les quartiers concernés, un changement de dimension et d'intensité de l'action publique, notamment par la perspective d'une stratégie de cohésion sociale et territoriale. Il s'agit d'améliorer les conditions de vie des habitants, de promouvoir l'égalité des chances et de marquer en profondeur et de manière durable, la transformation d'image et de perception des quartiers. Il s'agit également de redonner une valeur économique à ces territoires par des actions de revitalisation ${ }^{4}$. Dans les GPV, le sens ${ }^{5}$ du territoire est élargi par la prise en compte globale des dimensions sociales, économiques et politiques. D'ailleurs, les premiers éléments d'évaluation des GPV (Bansept, Crepey, Roussel, 2001) en fournissent de multiples illustrations. Les territoires prioritaires de la politique de la ville sont décrits selon des indicateurs non seulement urbains, mais aussi socio-économiques, administratifs et politiques : habitat dévalorisé, structures monolithiques sans diversité, insuffisance ou inadaptation des transports en commun, etc., mais aussi absence de politique directrice et de lignes structurantes, concentrations de population à forte densité de handicaps, limites du cadre bâti, sous-équipement en logement social, démographie en forte augmentation avec une insuffisance d'offre de logement à bas prix. Ces territoires sont des espaces où le taux de chômage dépasse largement la moyenne nationale, où le taux de RMIstes est très important et où près de la moitié, sinon davantage, d'allocataires $\mathrm{CAF}^{6}$ vivent en dessous du seuil de pauvreté. Les références au déficit structurel de l'emploi et au nombre restreint d'offres d'emploi en périphérie, comme d'ailleurs les références aux carences de mises en réseau des structures d'insertion et de qualification, de formation, ou au tissu commercial fragilisé et en régression, sont déterminantes pour des territoires faisant l'objet des GPV. Ici la dégradation de l'image de la ville n'est pas uniquement une question d'architecture, mais aussi d'insécurités sociales, économiques, sanitaires, scolaires, de violence et de délinquance. Il s'agit de territoires qui "décrochent» avant tout par le vécu des habitants, de territoires qui se démarquent par la précarisation et la paupérisation. Les GPV, dans leurs intentions et orientations, leurs actions urbaines et sociales :

Veulent aller vers une ville capable de faire société, une ville qui manifeste physiquement sa cohésion, par l'affirmation des continuités spatiales et l'élimination des coupures, où la cohésion sociale et le bien-être des gens sont recherchés en combinant l'action spatiale et l'action sociale, et où en outre, le lien social et la vie collective sont promus non en opposition mais en synergie avec l'épanouissement personnel (Bansept, Crepey, Roussel, 2001). 
Dans ce cadre, celui des GPV, l'intervention politique sur les territoires concernés, en raison de sa dimension globale, ne peut plus reposer uniquement ou principalement sur la référence au symbolique. Pour les GPV, le territoire n'est pas simplement un découpage dans l'espace de la ville, un quartier, un ensemble de lieux à "revisiter», mais à « rendre siens » aussi bien par les habitants que par la ville elle-même. Par conséquent, les délimitations politiques des territoires en question reposent, bien entendu, sur des études et sur des constats scientifiques, mais, en même temps, elles ne peuvent faire abstraction de «ce qui dans l'homme se dérobe au discours scientifique et frôle l'irrationnel, car le territoire est vécu, affectivité, subjectivité ou bien le nœud d'une religiosité» (Bonnemaison, 1981, p. 261). La production du symbolique dans un milieu de vie est d'ailleurs le premier indice de la territorialité : un milieu défavorisé au même titre qu'un «milieu professionnel peut être un territoire » (Barel, 1982). La territorialité a pour base dans les GPV, au-delà de la matérialité d'un fragment de l'espace géographique de la ville, «des territoires abstraits» (Raffestin, 1982), ceux produits par l'esprit humain. Le renouvellement des territoires est ainsi une réalité qui s'érige avant tout dans les lieux accueillant la projection des formes symboliques (Cassirer, 1972) sur l'espace. Ainsi apparaissent deux «hauts lieux» (Brunet, Ferras, Théry, 1992) tout particulièrement ouvert à l'« artisanat " du symbolique ${ }^{7}$ en contexte GPV : la contractualisation et la concertation.

\section{La contractualisation: symbolique d'appartenance au territoire}

6 En lançant un programme qui comprend $50 \mathrm{GPV}$ et 40 Opérations de Renouvellement Urbain (ORU) $)^{8}$, le CIV du 14 décembre 1999 a introduit la notion de renouvellement urbain'. Le concept de renouvellement urbain comporte avant tout la réactivation de l'appartenance d'un territoire déterminé à une ville par la référence à la mémoire de la ville en termes de vécus, perceptions, identités, comportements, représentations, etc. Il ne s'agit pas de qualifier, c'est-à-dire d'attribuer une qualité à un territoire afin qu'il devienne ville ou qu'il puisse appartenir à la ville. Il est question de projeter sur ce territoire la mémoire de son appartenance passée à la ville et en même temps accueillir dans un cadre précis la production symbolique déterminant la territorialité sensible. Les deux versants de la démarche sont complémentaires et indissociables du point de vue opérationnel. D'une part, la ville se « rappelle » que tel ou tel quartier lui appartenait à un moment donné et en vertu de cette appartenance historique, car inscrite dans le temps, elle entend se donner les moyens pour le «rendre sien ». D'autre part, par les mêmes productions symboliques de la mémoire, la ville rappelle au territoire en rupture que la fracture est justement l'indice d'une appartenance antérieure à prendre en compte afin de reconstruire la continuité originelle. En second lieu, le concept de renouvellement urbain renferme la dimension d'appartenance comme rapport entre un territoire et une ville par l'essence même de l'urbain dont il participe. Trois types de scénarios sont possibles : les scénarios où l'essence de l'urbain est saisie en termes physiques, de schéma d'utilisation des sols, de densité démographique, etc., les scénarios où l'essence de l'urbain est approchée en termes de «qualité et diversité de vie, aspects distincts et dans une certaine mesure indépendants" des caractéristiques retenues dans le type de scénario évoqué précédemment et, enfin, les scénarios où l'essence de l'urbain englobe l'ensemble des catégories précédentes. Mais quel que soit le type de scénario, c'est-à-dire 
la traduction dans la quotidienneté de ce concept, le fait même de rattacher une référence qualitative à un processus, en l'occurrence au renouvellement, induit inéluctablement une restriction dont la symbolique ne peut pas manquer de distinguer des appartenances afin de mettre en évidence des frontières et par conséquent des territoires. Ceci dit, la symbolique de l'appartenance à un territoire est peu active dans les GPV à partir du concept d'urbanisme lui-même ou de sa transposition effective. Deux raisons majeures semblent réunir les principaux éléments de réponse : l'obsession de la matérialité et le rôle de l'urbanisme dans l'approche globale des territoires engagée par la politique de la ville. Par exemple, la requalification des territoires faisant l'objet des GPV est systématiquement réduite à la "requalification des espaces extérieurs", «requalification d'un cœur de quartier», «requalification des quartiers", " requalification des voiries structurantes », « requalification des espaces publics ${ }^{10}$, etc. Quant au rôle de l'urbanisme dans le dispositif, il confirme une tendance déjà observée, c'est-à-dire celle de chercher à agir sur les processus sociaux et, en particulier, sur les processus d'interactions, par le biais des aménagements spatiaux, alors que ni les plans des villes traditionnelles ni les études qui les sous-tendent n'ont réussi à représenter la ville comme un processus opérant dans l'espace (Webber, 1996, p. 41).

Mais le concept de renouvellement urbain désigne bien plus qu'un changement de nature discursive permettant politiquement d'orienter le débat, c'est-à-dire l'échange éminemment langagier sur la ville. Il marque une nouvelle manière de déployer la politique de la ville dans l'espace public. Ces projets et opérations qui prennent le relais des GPU et qui permettent d'associer cette fois la dimension fonctionnelle à la dimension physique sont inscrits désormais dans les contrats de ville 2000-2006. Ainsi, les territoires concernés par les GPV, avant d'appartenir à la ville matérielle, appartiennent à la ville normative et symbolique. Il s'agit d'un processus de contractualisation où le concept de renouvellement urbain inscrit l'appartenance des territoires GPV au contrat de la ville en s'appuyant sur deux champs de productions symboliques : celui du texte et celui de la vie quotidienne. Le contrat sous sa forme visible étant texte et par conséquent écriture, il a la " possibilité de construire un espace conforme à un vouloir " (Certeau, 1990, p. 284) et le vouloir dans le cas de l'inscription du GPV dans le contrat de ville est suscité et entretenu par la conscience de l'appartenance à une territorialité commune. L'outil de contractualisation, notamment le contrat de ville, devient par conséquent procédure symbolique «d'animation et de coordination de l'ensemble des politiques publiques de lutte contre l'exclusion et d'intégration des populations et des ensembles urbains ${ }^{11}$. L'effet de l'appartenance à une territorialité commune apparaît alors en termes d'enjeux et d'analyses, c'est-à-dire en termes de productions symboliques et non pas simplement en termes de juxtaposition de produits financiers. À ce champ de production symbolique dont participe l'appartenance des territoires GPV aux territoires de la ville s'ajoute celui de la vie quotidienne ${ }^{12}$ des habitants rassemblant emploi, éducation, santé, droit, etc., car le renouvellement urbain est aussi revitalisation économique et renforcement du lien social, exercice de la citoyenneté, présence et qualité des services publics, développement de la justice de proximité, accès au droit, etc. L'inscription du GPV dans le contrat de ville ne restreint pas le sens de l'appartenance à une territorialité. Le GPV devient, au contraire, le lieu ${ }^{13}$ privilégié pour développer la contractualisation. Les différents aspects de la vie quotidienne constituant des territoires GPV renforcent la symbolique de l'appartenance à la ville en produisant des nouveaux contrats spécifiques. L'une des plus fortes illustrations est fournie par le rapport rendu au ministre délégué à la ville en avril 
2002 : «Pour des grands projets d'éducation dans les grands projets de ville» (Leydier, Mouchard-Zay, Mourlet, 2002). En effet, l'aspect éducation de l'appartenance à la territorialité de la ville active dans le processus de contractualisation les productions symboliques de l'animation, du sport, de l'enfance, de la jeunesse, de la formation, etc. Dans un sens, à ce niveau de la contractualisation, c'est le soutien à la parentalité, l'accès au savoir et les apprentissages, l'action péri et extra-scolaire, l'alphabétisation et la lutte contre l'illettrisme qui focalisent les symboliques d'appartenance aux territoires GPV. Dans l'autre sens, la symbolique de l'appartenance se construit autour de la citoyenneté, de l'autonomie, du partenariat, de la modernisation, de l'accompagnement scolaire, etc. La contractualisation apparait, donc, non seulement comme une symbolique de l'appartenance, mais aussi comme une condition pour la production de cette symbolique.

\section{La concertation : symbolique d'appropriation de territoire}

8 L'effet premier du renouvellement urbain est la recomposition des territoires de la ville. La notion de recomposition appliquée à celui de territoire, avant d'être utilisée dans l'élaboration des politiques de la ville, s'est imposée dans le discours des géographes. Ces derniers y sont parvenus après un cheminement épistémologique au but duquel apparait la nécessité de saisir le territoire à l'aide d'un concept capable d'interroger la volatilité et l'instabilité quasi permanente des espaces géographiques dans leur complexité physique, culturelle, sociale, politique, économique, etc. Il s'agissait de répondre à une problématique nouvelle, celle de l'« organicité évolutive » (Glon, Gonin, Gregoris, Renard, 1996, p. 196) du territoire, par rapport à laquelle le concept d'aménagement montrait ses limites. À ce moment, le concept de "recomposition» ouvre la voie à l'approche résolument phénoménologique du territoire. La recomposition focalise ainsi sur le territoire les projections symboliques de deux perspectives.

La première fait référence «à une période antérieure, de graves dysfonctionnements, voire de ruptures dans l'organicité au point d'entrevoir la désagrégation, la dissociation ou même la séparation et la division en éléments distincts, synonyme de décomposition » (ibid., p. 197).

La seconde, évoque "la possibilité d'effacer les stigmates d'une période bouleversante et déstructurante pour revenir à une composition antérieure. En relevant le risque insidieux d'un retour en arrière sur une situation idéale mythifiée, la notion de recomposition entretient la nostalgie bucolique, voire utopiste, accrue par les vertus salvatrices qu'on veut bien lui prêter » (ibid.).

Dans ce cadre, la recomposition comme symbolique de l'appropriation des territoires a été mise en évidence dans les études sur les mutations économiques (ibid., pp. 195-210). Ces approches montrent que la recomposition projette sur un territoire la production symbolique de l'« initiative » économique, tout en précisant que ce n'est pas la nature en elle-même de l'initiative qui est déterminante, mais la localisation de celle-ci dans l'espace. Plus exactement, l'initiative locale, dans la mesure où elle parvient à se faire identifier comme telle dans l'espace local, est investie d'une crédibilité constructive, c'est-à-dire, les opportunités pour une dynamique locale du territoire s'ouvrent dans la mesure où préalablement il $\mathrm{y}$ a eu une appropriation de l'initiative en question. En somme, la recomposition, avant de s'inscrire sur un sol et de produire une image dans la matérialité d'un lieu, repose sur des participations inégales, des interrelations et des 
échanges fortement symboliques dont le rôle est d'œuvrer à l'appropriation non pas d'un projet, mais d'un territoire « organique » avec des multiples facettes.

Dans les GPV, la recomposition des territoires concernés, dans le sens d'appropriation de ces territoires par ceux qui y habitent, mais aussi par la ville comme domaine administratif, présence physique et enchevêtrement immatériel d'interactions, se joue au niveau de la fonction symbolique du territoire dans la concertation. Il faut souligner que la concertation est une composante privilégiée dans tous les GPV mis en place. Le problème est de savoir si les promoteurs des GPV font appel à la concertation par souci de répondre à une demande de "surplus » de démocratie, pour se conformer à une exigence normative, afin de «faire passer » un projet, etc., ou comme moyen capable de favoriser l'appropriation des territoires symboliques, celui de la démocratie, celui de la liberté, celui de la responsabilité, celui de la sécurité, etc. Souvent les acteurs de l'action publique, en s'appuyant, sans doute, sur certaines études ${ }^{14}$, confèrent à la concertation l'aptitude de légitimer des projets. Mais cette aptitude est illusoire, car la concertation n'élimine pas la contestation. D'autres, surtout les habitants, notamment les représentants associatifs, s'emparent de la concertation avec le sentiment de participer à la décision. Participation trompeuse, car il est bien connu que la décision, par sa dimension de choix en matière de politique locale, revient toujours en termes de légitimité démocratique aux instances issues du suffrage universel. Les GPV permettent d'observer d'autres « vertus » confiées à la concertation. L'analyse d'un premier ensemble de GPV en 2001 souligne l'efficacité de la concertation et son rôle dans la déclinaison des détails des projets. Ainsi il y a « les GPV où l'on croit à l'efficacité de la concertation » non seulement pour l'élaboration des projets de proximité mais aussi dans le cadre de comités de pilotage, pour l'élaboration de la conduite d'un projet d'ensemble. Ensuite, il y a « les GPV où l'on privilégie la participation à la préparation de projets très précis et locaux " dans le cadre de réunions où élus et techniciens travaillent avec les habitants. Dans ce dernier cas, la concertation conduit à des procédures telles que la mise en place de fonds spécialisés pour permettre aux habitants de financer de petits aménagements de proximité selon leurs choix ainsi que des projets concernant la vie de quartier. Ces deux orientations ne sont pas exclusives et sont parfois développées de manière simultanée (Bansept, Crepey, Roussel, 2001). Il s'agit de traductions in situ de l'appropriation des territoires, dans un lieu de production symbolique, la concertation, elle-même forme symbolique de l'action collective (Bratosin, 2001).

\section{Conclusion}

11 Le programme GPV met en évidence une nouvelle orientation dans la considération politique du rôle joué par la dimension symbolique du territoire lorsqu'il est question de renouvellement urbain. D'abord, le passage de la référence au symbolique à la production du symbolique marque un changement fondamental concernant le sens donné aux territoires dans les GPV. Avant d'être matérialité spatiale, les territoires GPV sont des objets construits de l'esprit humain. Ce n'est plus à la construction en béton, fer, verre, etc., qu'est octroyée premièrement l'aptitude de fonder le sens d'un territoire transformé, mais à la construction symbolique d'une image à projeter sur l'espace à renouveler. En second lieu, l'inscription du projet concernant un territoire urbain dans le contrat de ville souligne que la substantialité du changement - entendue et attendue comme apport capable d'améliorer immédiatement les conditions de vie des habitants et de marquer en 
profondeur et de manière durable la transformation d'image et de perception d'un quartier - n'est efficace que dans la mesure où elle repose sur une reconnaissance préalable mutuelle d'appartenances symboliques aux territoires en question. Enfin, la recomposition des territoires GPV, puisqu'elle repose sur des appropriations des territoires en termes de produits symboliques de revalorisation économique et de revitalisation sociale, désigne la concertation comme lieu privilégié pour fournir aux formes symboliques l'opportunité d'une émancipation citoyenne en contexte procédural de légitimation démocratique.

\section{BIBLIOGRAPHIE}

- Circulaire du 27 décembre 2000 relative aux Grands Projets de Ville - problèmes d'enclavement des quartiers ou d'habitat privé dégradé, Paris, Ministère de l'emploi et de la solidarité.

- Contrat de ville. Pour un développement maîtrisé de l'agglomération toulousaine, Toulouse, novembre 2000.

- Des Grands Projets Urbains aux Grands Projets de Ville, Paris, DIV, décembre 1999.

- Identités des GPV - Deuxième rencontre nationale des Grands Projets de Ville, Paris, DIV, janvier 2002.

- Loi n²000-1208 du 13 décembre 2000 relative à la solidarité et au renouvellement urbains.

Bansept, C., Crepey, G. et Roussel, F.-X., (2001), « Rapport introductif : analyse d'un premier ensemble des GPV », in : Cahiers du Conseil National des Villes, Paris, juin.

Barel, Y., (1982), Le social et ses territoires, Actes du colloque « Géopoint 82 », Avignon.

Bonnemaison, J., (1981), « Voyage autour du territoire », L'Espace Géographique, Paris.

Bratosin, S., (2001), La concertation : forme symbolique de l'action collective, Paris, l'Harmattan.

Brunet, R., (1967), Les phénomènes de discontinuité en géographie, Paris, CNRS, 1967.

Brunet, R., Ferras, R. et Théry, H., (1992), Les mots de la géographie, Paris, Reclus/La Documentation française.

Cassirer, E., (1972), La philosophie des formes symboliques, Paris, Minuit.

Certeau, M. de, (1990), L'invention du quotidien. Art de faire, Paris, Gallimard.

Cioran, E., (1995), Euvres, Paris, Gallimard.

Fourniau, J. M., (1996), « Les décisions d'infrastructures soumises au débat public », in : Métropolis, n¹06-107.

Gay, J.-C., (1995), Les discontinuités spatiales, Paris, Economica.

Glon, E., Gonin, P., Gregoris, M. T. et Renard, J. P., (1996), « Mutations économiques et recompositions des territoires », in : Nord/Pas-de-Calais : changement régional et dynamique des territoires, Lille, FRE-Ville.

Leydier, J., Mouchard-Zay, H. et Mourlet, E., (2002), « Pour des grands projets d'éducation dans les grands projets de ville », Rapport rendu au ministre délégué de la ville, Paris, avril. 
Raffestin, C., (1982), Les territoires de la vie quotidienne, Actes du colloque « Géopoint 82 », Avignon.

Ricœur, P., (1986), Du texte à l'action, Paris, Seuil.

Sfez, L., (1992), Critique de la décision, Paris, Presses de la Fondation Nationale de Sciences Politiques.

Webber, M. W., (1996), L'urbain sans lieu ni bornes, Paris, l'Aube.

\section{NOTES}

1. Élément de base pour les modèles spécifiques d'organisation de l'espace en raison de son ambivalence, à la fois synapse et cloison, interface et barrière. À distinguer de la « coupure » qui n'est pas un lieu qui participe de l'urbanité, même si l'on attache souvent à ce lieu une fonction d'intérêt général.

2. Cf. Des Grands Projets Urbains aux Grands Projets de Ville, Paris, DIV, décembre 1999.

3. Par «symbolique » il faut entendre ici la prolongation phénoménologique du symbole dans le discours.

4. Cf. Circulaire du 27 décembre 2000 relative aux Grands Projets de Ville - problèmes d'enclavement des quartiers ou d'habitat privé dégradé, Paris, Ministère de l'emploi et de la solidarité.

5. Le «sens» comme projection de l'agissement humain (voir Cioran, 1995, p. 1782), ici concernant la territorialité urbaine.

6. Caisses d'Allocations Familiales.

7. L'« artisanat » du symbolique ou l'activité de production et/ou de transformation des discours fondateurs.

8. Un second CIV, en octobre 2001, ajoute 30 nouvelles ORU à ce programme.

9. La notion de "renouvellement urbain " sera reprise un an plus tard par la loi SRU (loi ${ }^{\circ}$ 2000-1208 du 13 décembre 2000). Cette loi est un vaste chantier législatif qui apporte des changements dans de nombreux textes normatifs : le code de l'urbanisme, le code de la construction et de l'habitation, la loi du 10 juillet 1965 sur la copropriété, la loi du 6 juillet 1989 sur les rapports locatifs, le code civil, le code de la santé publique, etc. Cependant, les concepts essentiels de la loi SRU sont remis aujourd'hui en question par la loi du 2 juillet 2003 « urbanisme et habitat »: le régime juridique des schémas de cohérence territoriale et des plans locaux d'urbanisme sont substantiellement modifiés.

10. Voir Identités des GPV - Deuxième rencontre nationale des Grands Projets de Ville, Paris, DIV, janvier 2002.

11. Contrat de ville. Pour un développement maîtrisé de l'agglomération toulousaine, Toulouse, novembre 2000.

12. Approche absolument pertinente dans la mesure où l'on considère que le texte et l'action sensée participent du même paradigme (voir Ricœur, 1986, pp. 205-236).

13. «Est un lieu l'ordre (quel qu'il soit) selon lequel des éléments sont distribués dans des rapport de coexistence » (Certeau, 1990, pp. 172-173).

14. Voir par exemple Fourniau, 1996. 


\section{RÉSUMÉS}

Alors que le programme politique des GPV rattache le renouvellement urbain à une reconsidération globale des territoires de la ville, cet étude met en évidence l'émergence du territoire urbain comme lieu d'une véritable production symbolique. Une approche comparative avec le programme des GPU fait apparaître ainsi dans la démarche politique le passage de la référence aux symboliques des territoires à la production d'une symbolique des territoires. Lieux révélateurs de cette production, les dispositifs techniques et politiques de contractualisation et de concertation permettent de saisir les indices de cette nouvelle manière de susciter ou de modeler le territoire urbain: d'une part, la contractualisation réactive la fonction d'une symbolique d'appartenance au territoire, d'autre part, la concertation fait ressurgir une symbolique d'appropriation du territoire comme enjeu de l'aménagement.

Whereas the political program of the Major City Projects (GPV) program connects urban renewal to a global reconsideration of the territories of the city, this study shows the emergence of the urban territory as a place of symbolic production. A comparison with the Major Urban Projects (GPU) program reveals in the political method the shift from mere reference to the symbolic of the territories to the production of a symbolic of the territories. Revealing places of this production, the technical and political mechanisms of contractualization and dialogue make it possible to capture the indicators of this new approach to creating or modeling the urban territory. While contractualization reactivates the function of a symbolic of belonging to the territory, dialogue leads to a re-emergence of a symbolic of appropriation of the territory as a challenge in urban development.

\section{INDEX}

Mots-clés : urbanisme, aménagement du territoire, symbolique

Keywords : town planning, country planning, symbolic

\section{AUTEUR}

\section{STEFAN BRATOSIN}

Cerass - Université de Toulouse 3 